\title{
Final year medical students versus interns: information seeking behaviour about COVID-19 therapy in India
}

\author{
Rosme David $^{1 *}$, Deepthi Mary George ${ }^{1}$, Shalini Chandra ${ }^{2}$, \\ Vishal Marwaha ${ }^{3}$, Princy Louis Palatty ${ }^{1}$
}

\author{
${ }^{1}$ Department of Pharmacology, Amrita Institute of Medical Science, Kochi, Kerala, India \\ ${ }^{2}$ Department of Pharmacology, Rohilkhand Medical College and Hospital, Bareilly, Uttar Pradesh, India \\ ${ }^{3}$ Department of Rheumatology, Amrita Institute of Medical Science, Kochi, Kerala, India
}

Received: 25 February 2021

Revised: 06 April 2021

Accepted: 09 April 2021

*Correspondence:

Dr. Rosme David,

Email: ed.rosme@gmail.com

Copyright: (C) the author(s), publisher and licensee Medip Academy. This is an open-access article distributed under the terms of the Creative Commons Attribution Non-Commercial License, which permits unrestricted non-commercial use, distribution, and reproduction in any medium, provided the original work is properly cited.

\begin{abstract}
Background: Doctors alone must be capable of taking ultimate responsibilities for making decisions in clinical uncertainties. A right clinical judgement and management was the ultimate priority for health care workers during the COVID-19 pandemic. The objective of our study was to access knowledge about COVID-19 treatment among the final year bachelor of medicine and bachelor of surgery (MBBS) students and interns and thereby to understand the information seeking behaviour.

Methods: Multicentric cross-sectional questionnaire-based study among the final year MBBS students and interns. The google form questionnaire was sent to the participants through whatsapp or mail. The questions were related to the drugs, the precautionary measures and the dead body disposal in COVID-19. Attitude regarding seeking information about the new disease, updated treatment guidelines as well as the preferred resource materials was also studied. The sample size was calculated based on a pilot study.

Results: Out of 316 participants, 30.7\% had good, 53.2\% had adequate and $16.1 \%$ had inadequate knowledge regarding the updated treatment guidelines. In one of the questions about hydroxychloroquine, 51.5\% final year MBBS students $(n=200)$ and $63.8 \%$ interns $(n=116)$ responded correctly $(p<0.034) .65 .4 \%$ gathered information by self-directed learning through various sources. $45.8 \%$ gathered information from social media while $44.4 \%$ read printed materials and $39.3 \%$ heard online/offline lectures.

Conclusions: We conclude that the final year MBBS students and interns have satisfactory knowledge about COVID19 treatment. Interns had better awareness than the final year MBBS students. Retaining the theoretical knowledge during internship will make the young doctors more confident while practicing.
\end{abstract}

Keywords: COVID-19, Final year, Interns, Knowledge, Information seeking behaviour

\section{INTRODUCTION}

In December 2019, in the city of Wuhan, China, an outbreak of an emerging disease COVID-19 due to a novel coronavirus, later named as SARS-CoV-2 was dectected. ${ }^{1,2}$ On March 2020, WHO declared this epidemic of COVID-19 as a pandemic. ${ }^{3}$ COVID-19, a novel pandemic was claiming many lives including healthcare workers is an imminent field to take action to save lives including doctors. As of now no specific treatment protocol has been found for the treatment. ${ }^{4,5} \mathrm{We}$ are gaining disseminated knowledge by trial-and-error 
strategy and a conjecture is being used. Amongst the healthcare professionals, it is always the doctors who should take ultimate responsibilities for difficult decisions in situations of clinical complexities and uncertainties, drawing on their scientific knowledge and well-developed clinical judgement. The total cases have come to a plateau phase and now we dwell on the fear of a second wave due to mutated viruses. After a year, the reports say, 10 crore people had been infected in the whole globe with 21 lakh deaths. The global situation is reflecting in our populated country too. 1 crore Indians had the disease, of which 1.5 lakh died due to COVID-19.

In a descriptive study by Khasawneh et al at Jordan, among medical students, it was found that the students showed expected levels of knowledge and attitude regarding COVID-19 and took good precautionary measures. They also concluded that in the current global situation there is a need for frequent utilization of social media by medical schools to impart knowledge. ${ }^{7}$ Similarly, a cross sectional study conducted by Joshi et al in India about the knowledge, attitude and practices regarding COVID-19 among medical students, $94.15 \%$ of the medical students had extensive knowledge of the COVID-19 pandemic. The study also proved that there is a clear need for regular orientation and training programs to improve and update knowledge regarding COVID-19 infections and prevention strategies. ${ }^{8}$ Our study aims at exploring the knowledge about the current treatment modalities of COVID-19, among the final year medical students and interns across the country. The knowledge about the proper precautionary measures, dead body disposal and the information seeking behaviour among the students is also being evaluated.

\section{Aim}

The aim of this study was to understand and compare the knowledge about COVID-19 therapy among final year MBBS students and interns across the country.

\section{Objectives}

The primary objective of this study was to assess the knowledge about COVID-19 therapy among final year MBBS students and interns and to compare the knowledge about COVID-19 therapy between final year MBBS students and interns.

The secondary objective of this study was to assess the student's interest to be updated with the latest treatment guidelines and preferred resource for updation.

\section{Rationale and relevance}

The world braces for the COVID-19 pandemic and healthcare workers on the frontlines are particularly vulnerable to this infection. Discussions and research are taking place worldwide because it is a disease not only affecting the health of the people but also affecting every aspect of an individual including mental health, financial fields and economy. Our study helps us to know, how vigilant are our young doctors to know about the updated COVID-19 treatment protocols and the precautionary measures they practice themselves to serve the community.

\section{METHODS}

It was a multicentric cross-sectional questionnaire-based study among final year MBBS students and interns across India. The google form 20 item questionnaire was sent through social media. The data was analysed by the principal investigator at Amrita institute of medical science, Kochi.

\section{Study tools}

The validated multiple option questionnaire was prepared in the google form. The questions were self-designed by the investigators after referring standard pharmacology textbooks and was validated. The WHO and Centre for Disease Control and Prevention (CDC) updated treatment guidelines for COVID-19 was also referred.

The google form comprised of 5 sections, which takes about 5-10 min to complete. The participant information sheet was in the first section. The second section had informed consent. Those who agreed to give consent will proceed to the third section. This section had the 20 -item quiz. Of the 20 questions, 5 were regarding precautionary measures, 2 were related to dead body disposal and disinfecting premises. The remaining 13 questions were related to drugs of which 2 questions were on antibiotics. Those who answered correctly, received a score 1 for each question. The fourth section had 4 qualitative open-ended question about their information seeking behaviour and the last section was to choose their category as final year MBBS student or intern. After clicking the submit button, the participants received their total score out of 20 with the correct responses and explanation.

\section{Study duration}

The study was conducted from August 2020 to December 2020. The google form was sent through social media for data collection from 17 August 2020 to 9 November 2020.

\section{Consent}

The consent was taken through the google form.

\section{Sample size}

Since there is no other study done to assess and compare the knowledge about COVID-19 therapy among final year MBBS students and interns, we conducted a pilot study with 16 participants. From that study report we estimated the minimum number of sample size as 300 for the main study. 


\section{Data analysis}

All the collected data were entered into microsoft excel 2020 and cross checked for presence of any error to maintain its accuracy. Qualitative data were represented as descriptive statistics. Chi-square test was used to investigate the level of association among study variables. A $\mathrm{p}$ value of less than 0.05 was considered statistically significant. Statistical analysis was performed using IBM SPSS version 16

\section{RESULTS}

Among the 316 participants, $63.3 \%$ were final year MBBS students and $36.75 \%$ were interns. The excel data was then uploaded in SPSS software version 16 and used the descriptive statistics, Pearson chi-square test and Levene's test for analysing and representing the data.

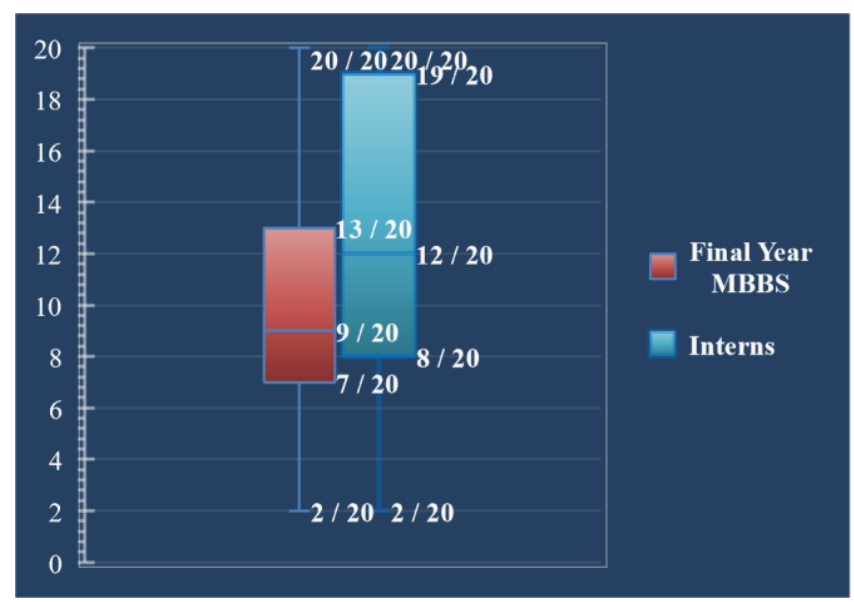

Figure 1: Box and Whisker plot for the scores among final year MBBS students $(n=200)$ and interns $(n=116)$.

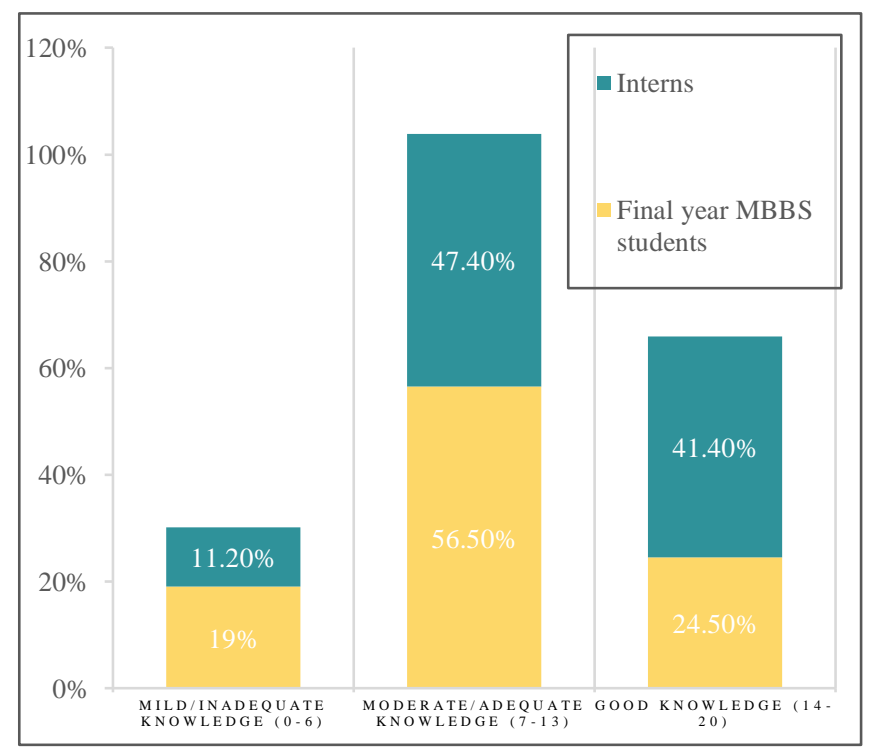

Figure 2: Comparison of knowledge among final year MBBS students $(n=200)$ and interns $(n=116)$.

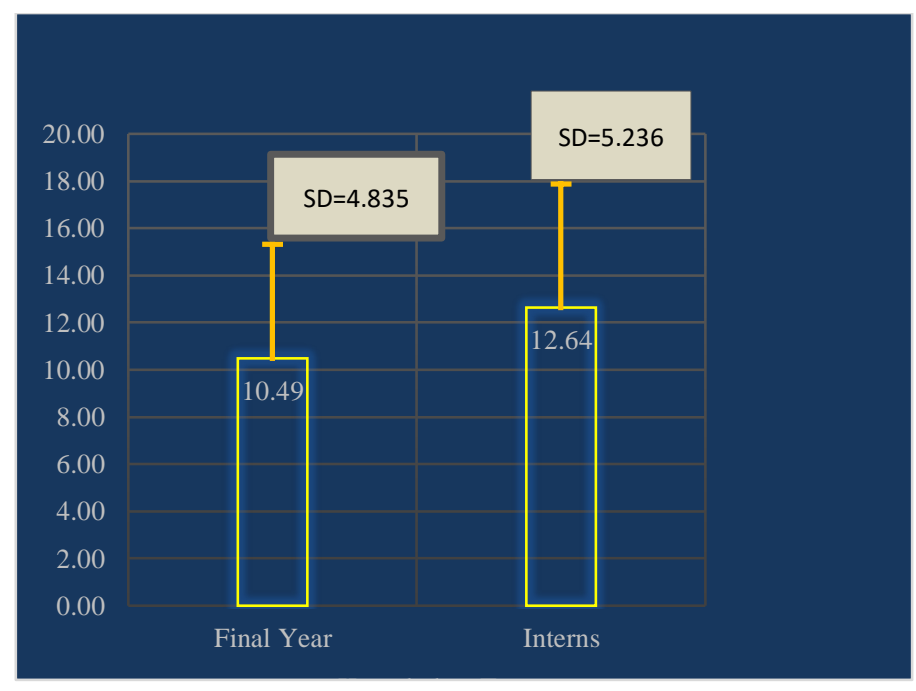

Figure 3: Mean score with standard deviation (SD) among final year MBBS students $(n=200)$ and interns $(n=116)$.

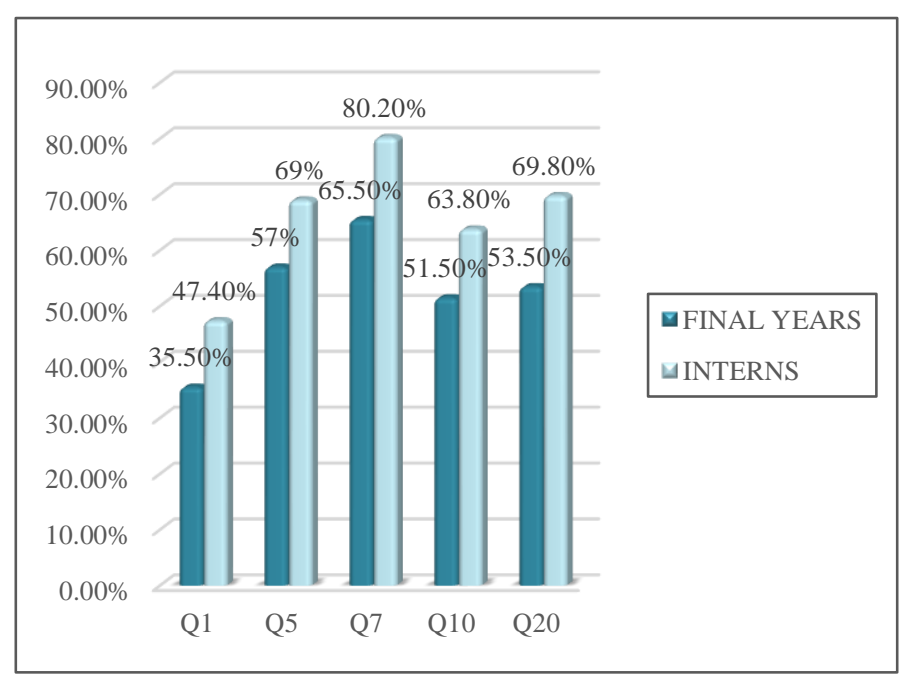

Figure 4: Comparison of correct answers among final year MBBS students $(n=200)$ and interns $(n=116)$.

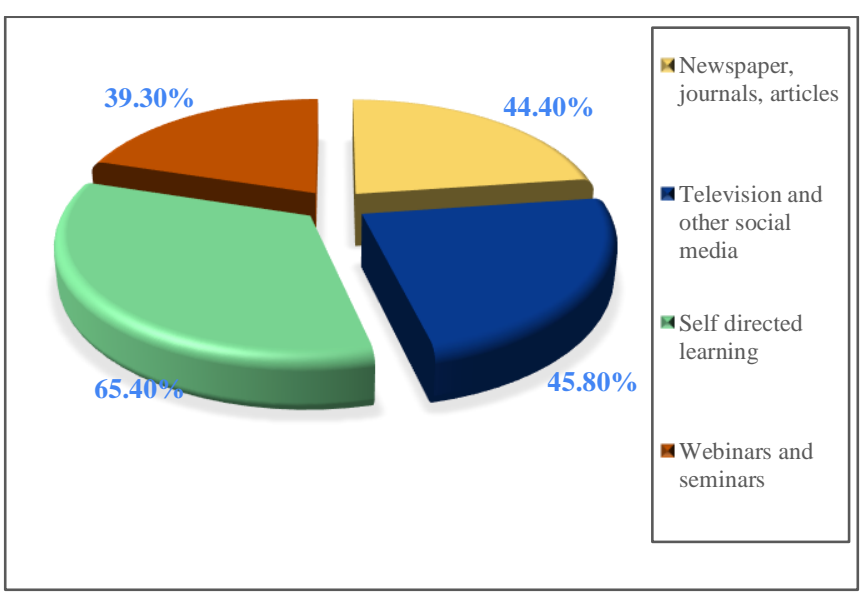

Figure 5: Preference of resources for updating $(n=295)$. 


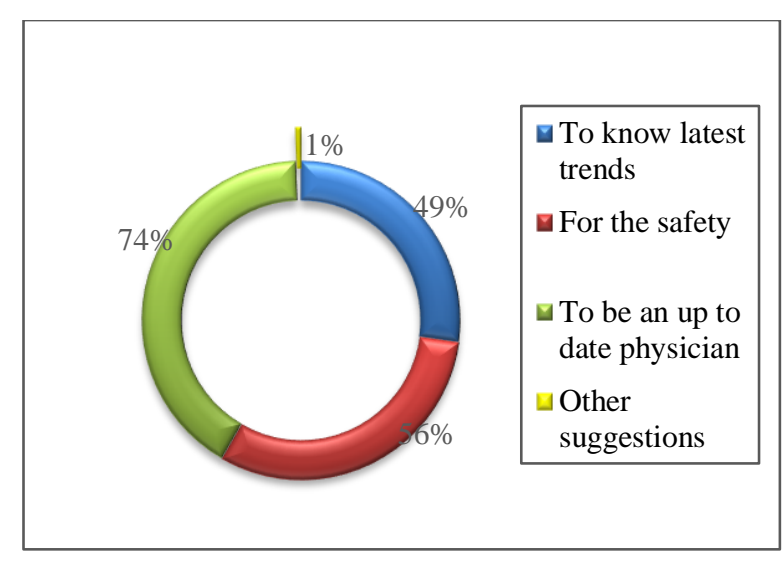

Figure 6: Reason for updating $(n=298)$.

A total 20 single response quiz was conducted through google form and for each correct answer they scored 1 point. The scores range from $2 / 20$ to $20 / 20$. The scores among the 2-study group were depicted in Box and Whisker plot (Figure 1).

Based on the scores obtained in the quiz, the participants $(n=316)$ were categorised into 3 groups: inadequate/mild knowledge, adequate/moderate knowledge and good knowledge category (Table 1). The categorised knowledge among the final year MBBS students and interns were depicted in Figure 2, which was statistically significant since the $\mathrm{p}$ value is $0.005(\mathrm{p}<0.05)$.

Regarding the use of antibiotic in coronavirus infection, $57 \%$ final year MBBS students $(n=200)$ and $69 \%$ interns $(\mathrm{n}=116)$ answered correctly $(\mathrm{p}<0.035) .36 .5 \%$ final year MBBS students $(n=200)$ and 53.4\%, 62 interns $(n=116)$ scored correctly $(\mathrm{p}<0.003)$ about antibiotic initiation (Figure 4).

With respect to the precautionary measures, $75 \%$ of final year MBBS students $(n=200)$ and $72.4 \%(n=116)$ interns correctly answered about the duration of hand wash. $71.5 \%$ and $81 \%$ of final year MBBS students $(n=200)$ and interns $(\mathrm{n}=116)$ respectively agreed on washing with soap and water as an effective method to prevent coronavirus infection.

The knowledge about COVID-19 disinfection and dead body burial was also analysed. The question on disinfection was answered correctly by $65.5 \%, 131$ $(n=200)$ final year MBBS students and 80.2\%, $77(n=116)$ interns. Pearson chi-square value was 0.006 ( $\mathrm{p}<0.05) .69 \%$ $(n=200)$ and $66.4 \%(n=116)$ of final year MBBS students and interns scored correctly for the question about disposal of dead body.

The dosage regimen of hydroxychloroquine for COVID-19 prophylaxis was analysed. 51.5\% $(\mathrm{n}=200)$ final year MBBS students and $63.8 \%(\mathrm{n}=116)$ interns responded correctly. Pearson chi-square value was $0.034(\mathrm{p}<0.05)$. Contraindication for hydroxychloroquine was correctly answered by $38.5 \%, 77(\mathrm{n}=200)$ and $64.7 \%, 75(\mathrm{n}=116)$ final year MBBS students and interns, respectively. Pearson chi-square test was $<0.001(\mathrm{p}<0.05)$.

Few questions were asked regarding the remdesivir therapy in COVID-19 treatment. For the basic pharmacological question about the antiretroviral classification, $61 \%$ final year MBBS students and $65.5 \%$ interns said correctly and about the regimen, 57\% final year MBBS students and $57.8 \%$ interns got right answer. $82 \%$ final year MBBS students and $60 \%$ interns agreed with elevation of hepatic enzyme as an adverse reaction of remdesivir therapy. eGFR being the recommended test before initiating the remdesivir therapy was agreed upon by $63 \%$ final year MBBS students and $72.4 \%$ interns. For assessing clinical knowledge about mandatory requirement for initiation of remdesivir treatment, 37.5\%, $75(\mathrm{n}=200)$ final year MBBS students and $60.3 \%, 70(n=116)$ interns answered correctly $(\mathrm{p}<0.001)$. For the question on contraindication of remdesivir, under emergency use authorization (EUA), $35 \%, 70(\mathrm{n}=200)$ final year MBBS students and 55.2\%, 64 $(\mathrm{n}=116)$ answered correctly $(\mathrm{p}<0.001)$.

Knowledge about the use of uncommon dugs like febuxostat, interleukins and anti-parasitic drugs in COVID-19 treatment was evaluated. For the question about febuxostat, 32.5\%, $65(n=200)$ final years MBBS students and $56.9 \%, 66(\mathrm{n}=116)$ interns answered correctly $(\mathrm{p}<0.001)$. About the knowledge on use of tocilizumab in COVID-19, 69\% final year MBBS students $(n=200)$ and $70.7 \%$ interns $(n=116)$ scored right and about antiparasitic drugs in the prophylaxis, 53.5\%, $107(\mathrm{n}=200)$ final year MBBS students and 69.8\%, $81(n=116)$ interns answered correctly $(\mathrm{p}<0.004) .93 .4 \%$ agreed yes-to be updated with COVID-19 treatment protocols with available resources (Figure 5). 94.3\% participants agreed yes-they wish to seek information about new disease. The rationale for the same was assessed (Figure 6).

Table 1: Number of participants $(n=316)$ in each category.

\begin{tabular}{|lll|}
\hline Knowledge category & $\begin{array}{l}\text { Scores obtained out of } \\
\mathbf{2 0}\end{array}$ & $\begin{array}{l}\text { Percentage }(\boldsymbol{\%}) \\
(\mathbf{n = 3 1 6})\end{array}$ \\
\hline Inadequate/mild & $<6$ & $16.1(51)$ \\
\hline Adequate/moderate & $7-13$ & $53.2(168)$ \\
\hline Good & $>14$ & $30.7(97)$ \\
\hline
\end{tabular}


Table 2: Percentage of correct and wrong answers among final year MBBS students and interns with Pearson chisquare test.

\begin{tabular}{|c|c|c|c|c|c|}
\hline & \multicolumn{2}{|c|}{$\begin{array}{l}\text { Final year MBBS students } \\
(\mathbf{n = 2 0 0 )}\end{array}$} & \multicolumn{2}{|c|}{ Interns (n=116) } & \multirow{2}{*}{$\begin{array}{l}\text { Pearson chi-square } \\
(p<0.05)\end{array}$} \\
\hline & $\begin{array}{l}\text { Correct } \\
(\%)\end{array}$ & $\begin{array}{l}\text { Wrong } \\
(\%)\end{array}$ & $\begin{array}{l}\text { Correct } \\
(\%)\end{array}$ & $\begin{array}{l}\text { Wrong } \\
(\%)\end{array}$ & \\
\hline Q1 & 35.5 & 64.5 & 47.4 & 52.6 & 0.037 \\
\hline Q2 & 75 & 25 & 72.4 & 27.6 & 0.613 \\
\hline Q3 & 71.5 & 28.5 & 81 & 19 & 0.059 \\
\hline Q4 & 64 & 36 & 62.9 & 37.1 & 0.849 \\
\hline Q5 & 57 & 43 & 69 & 31 & 0.035 \\
\hline Q6 & 36.5 & 63.5 & 53.4 & 46.6 & 0.003 \\
\hline Q7 & 65.5 & 34.5 & 80.2 & 19.8 & 0.006 \\
\hline Q8 & 69 & 31 & 66.4 & 33.6 & 0.630 \\
\hline Q9 & 32.5 & 67.5 & 56.9 & 43.1 & $<0.001$ \\
\hline Q10 & 51.5 & 48.5 & 63.8 & 36.2 & 0.034 \\
\hline Q11 & 38.5 & 61.5 & 64.7 & 35.3 & $<0.001$ \\
\hline Q12 & 61 & 39 & 65.5 & 34.5 & 0.424 \\
\hline Q13 & 57 & 43 & 57.8 & 42.2 & 0.895 \\
\hline Q14 & 41 & 59 & 51.7 & 48.3 & 0.065 \\
\hline Q15 & 37.5 & 62.5 & 60.3 & 39.7 & $<0.001$ \\
\hline Q16 & 63 & 37 & 72.4 & 27.6 & 0.088 \\
\hline Q17 & 35 & 65 & 55.2 & 44.8 & $<0.001$ \\
\hline Q18 & 69 & 31 & 70.7 & 29.3 & 0.753 \\
\hline Q19 & 35 & 65 & 42.2 & 57.8 & 0.200 \\
\hline Q20 & 53.5 & 46.5 & 69.8 & 30.2 & 0.004 \\
\hline
\end{tabular}

\section{DISCUSSION}

This study discusses the knowledge regarding updated COVID-19 treatment as well as the information seeking behaviour among final year MBBS students and interns. The overall score of the knowledge about COVID-19 treatment. A total of 316 participants answered the google form questionnaire of which $63.3 \%$ were MBBS students. This higher proportion of medical student participants could be because the students might be at their residence during the lockdown period and they had spare time for participating in research rather than interns who were busy with their posting. A similar study among medical students by Khalil et al about COVID-19 in Baghdad city showed that majority of participants were medical students. ${ }^{9}$

Taghrir et al survey among medical students in Iran, about COVID-19 reported high level of knowledge about the novel disease. ${ }^{10}$ In another COVID-19 knowledge related study by Joshi et al at Telangana state in India showed that 75.6\% participants had high level of understanding regarding the novel disease. ${ }^{8}$ A survey among medical and allied health care students across India found that the participants had adequate knowledge regarding the new disease. ${ }^{11}$ An online questionnaire based survey by Jose et al among the pharmacy students in Kerala showed that $>70 \%$ of the participants had good understanding about the transmissibility of coronavirus. ${ }^{12}$ These results from various studies showed that there is a good understanding about the transmission and precautionary measures about novel coronavirus infection among the medical students.
Our study results showed that the depth of knowledge among medical students and interns were only satisfactory. This difference can be attributed to the fact that our study assessed the knowledge regarding the treatment of COVID-19 infection rather than pathophysiology which were assessed in the other studies.

When we compare among the participants, interns scored $>14$ while most of the final year MBBS students scored between 7-13 score. This difference could be due to the reason that interns were in the frontline during the pandemic and they were learning as they worked. Even though the questions that were asked in this survey were not from the current MBBS curriculum, only $19 \%$ final years MBBS students and $11.2 \%$ interns scored $<6$ score in this study, which shows that medicos had an enthusiasm to learn about the new disease.

About the hand hygiene, $>70 \%$ participants in final year MBBS students and interns agreed that washing with soap and water is more effective than alcohol-based hand sanitizers as precautionary measure. In 2017 Pranav et al conducted a questionnaire-based survey about hand hygiene practices among medical undergraduate students in India. 68\% agreed that hand rubbing is not more effective than hand washing and only $36 \%$ knew that the exact time required for hand washing was 20 seconds. ${ }^{13}$ The study finding was comparable with study by Taghrir et al among medical students in Iran where $>85 \%$ agreed upon washing hands with soap and water as an effective method to prevent infection from coronavirus. ${ }^{10}$ Here in this study, 
the final year MBBS students and interns were equally right on the quantum of time required for hand washing. This could be due to fact that there is a vast advertisement through social media about 20 seconds hand wash to prevent coronavirus. The WHO recommended percentage of ethanol and isopropyl alcohol content in hand sanitizers was assessed (Q1 and Q4). Though the results were not satisfactory among both study group, their working knowledge is more important. ${ }^{14}$

The use of antibiotic in COVID-19 treatment was assessed and interns answered better than final year MBBS students because they were experienced and have seen the use of these drugs. But other studies outside India showed that antibiotics are not effective in the first line treatment for COVID-19 which was agreed by $63.3 \%$ medical undergraduate students in Lahore, Pakistan as well as $66.9 \%$ medical undergraduates in Bagdad city. ${ }^{9,15}$ In a study among medical and allied health science students by Gohel et al about COVID-19, 25\% thought antibiotics might be useful, which was considered a wrong perception, which is a debatable subjective issue as proposed by the investigators. ${ }^{11}$

More than two third of the participants from final years and interns had good knowledge regarding the dead body disposal. This indicates our students had sought information and were empowered with necessary knowledge.

In reference to hydroxychloroquine and remdesivir both the study groups had satisfactory understanding about classification, indication and adverse drug reaction. When we compared, interns had better clarity than the final year MBBS students regarding the contraindication as well as mandatory requirement for the initiation of abovementioned drugs. In 2017 Meghna et al conducted a comparative study in India where the final year MBBS students were pitted against interns about pharmacotherapeutics. It was found that interns had better awareness regarding cardiovascular-pharmacology, drugs in emergency use as well as chemotherapy. ${ }^{16}$ This was attributed to the application of knowledge by the interns.

Use of other drug was well answered by both final year MBBS students and interns. In a question regarding use of febuxostat, interns answered correctly with a difference of $25 \%$ than the final year MBBS students.

Self-directed learning was the preferred ways for final year MBBS students as well as interns. Social media was more accessed than printed materials or expert classes. This finding was consistent with other studies in India as well as other parts of world. A study among medical students in Jordan showed that they rely mainly on online resources (social medias and search engines) for gathering the information. ${ }^{7}$ Medias (television, online, newspaper) gave much exposure about the new diseases, as stated by Maheshwari et al study in India. ${ }^{17}$ Therefore, self-directed learning using different accurate resources like WHO or
CDC guidelines are the only way for the students of today, to learn about the new disease as there is no previous reference for emerging disease. Being in the confined environment of pandemic, students depend on social medias for easy access of information.

$74.5 \%$ opted to be an updated physician rather than gaining knowledge for personal safety $(56 \%)$. This is a good indicator of their inherent professionalism. In an openended question, one of the students wants to reach out the latest treatment options to the public as well. So, this means that the students are highly professionals and always wish to be an experienced clinician.

We note that the overall knowledge is satisfactory and same among the final year MBBS students and interns. A higher awareness and completeness were observed among the interns. This is expected due to the good academic grounding from in house training which is focussed on interns but not with the undergraduates to be an expert clinician. Similar results were also noted from Singh et al study in India where they emphasised the need of improvement among undergraduates about the generic medicines. ${ }^{18}$ In contradictory, a study done by Mira et al in 2016 in India about clinical pharmacology and rational therapeutics (CPT) among MBBS students and interns, stated that the undergraduate students have better score than interns. The rationale could be because theoretical teaching was given to undergraduate students but was not retained during internship. ${ }^{19}$

Although there are many studies conducted among medical students, health care professionals and various other categories, we could not find a study which assessed the COVID-19 treatment knowledge among young medical students and interns.

Our study had a few limitations. This was an online questionnaire-based survey, so the sample size need not directly represent the target population. Since limited number of questions were asked, we included only basic and relevant pharmacology related topics.

\section{CONCLUSION}

The final year MBBS students and interns are well versed with the knowledge about treatment of new disease COVID-19 but interns are more accurate because the interns had good foundation in basics of pharmacology and its extrapolation during internship. A new disease of different source and pathophysiology may emerge in future. Therefore, we should focus more intellectually while moulding young doctors to withstand every toughest situation with the power of wisdom and knowledge. Seeking apt, accurate and authoritative information is necessary for building-up the knowledge. Retaining basic pharmacotherapeutics while facing newer real scenarios will help them gain more confidence for the trial-and-error methods of success. 


\section{ACKNOWLEDGEMENTS}

We are so grateful for those who helped us to reach out to various medical institutions across India.

Funding: No funding sources

Conflict of interest: None declared

Ethical approval: The study was approved by the Institutional Ethics Committee

\section{REFERENCES}

1. Lai C, Shih T, Ko W, Tang H, Hsueh P. Severe acute respiratory syndrome coronavirus 2 (SARS-CoV-2) and coronavirus disease-2019 (COVID-19): The epidemic and the challenges. Int J Antimicrob Agents. 2020;55(3): 105924.

2. Wang L, Wang Y, Ye D, Liu Q. Review of the 2019 novel coronavirus (SARS-CoV-2) based on current evidence. Int $\mathrm{J}$ Antimicrob Agents. 2020;55(6):105948.

3. WHO. Fact sheet: WHO director-general's opening remarks at the media briefing on COVID-19 - 28 December 2020, 2020. Available at: https://www.who.int/director-

general/speeches/detail/who-director-general-sopening-remarks-at-the-media-briefing-on-covid-19--28-december-2020. Accessed on 14 January 2021.

4. Huang C, Wang Y, Li X, Ren L, Zhao J, Hu Y, et al. Clinical features of patients infected with 2019 novel coronavirus in Wuhan, China. Lancet. 2021;395:497506.

5. Fen O, Heyu W, Ying Y, Wei T, Jing Z, Jie G. Measures to respond to the rapid spread of novel coronavirus pneumonia-China knowledge base. Docu Knowl Net. 2020;13(19):37.

6. Worldometer. Fact sheet: Coronavirus update: 93,533,917 cases and 2,002,411 deaths from COVID19 virus pandemic. Available at: https://www.worldometers.info/coronavirus/.

Accessed on 15 January 2021.

7. Khasawneh AI, Humeidan AA, Alsulaiman JW, Bloukh S, Ramadan M, Al-Shatanawi TN, et al. Medical students and COVID-19: knowledge, attitudes, and precautionary measures. a descriptive study from Jordan. Front Pub Heal. 2020;8:253.

8. Joshi KP, Madhura L, Jamadar D. Knowledge and awareness among nursing students regarding the COVID-19: a cross sectional study. Int J Comm Med Public Heal. 2020;7(7):2518.

9. Khalil N, Al-Yuzbaki D, Tawfeeq R. COVID-19 knowledge, attitude and practice among medical undergraduate students in Baghdad City. Eur Asian J Biosci. 2020;4186:4179-6.
10. Taghrir MH, Borazjani R, Shiraly R. COVID-19 and iranian medical students; A survey on their relatedknowledge, preventive behaviors and risk perception. Arch Iran Med. 2020;23(4):249-54.

11. Gohel KH, Patel PB, Shah PM, Patel JR, Pandit N, Raut A. Knowledge and perceptions about COVID-19 among the medical and allied health science students in India: an online cross-sectional survey. Clin Epidemiol Glob Heal. 2021;9:104-9.

12. Kochuparambil J, Issac A, George S, Panicker N. Knowledge pertaining to COVID19 among medical population of Indian state of Kerala: An online crosssectional survey. J Patient Saf Infect Control. 2020;8(1):17-20.

13. Modi PD, Kumar P, Solanki R, Modi J, Chandramani $\mathrm{S}$, Gill N. Hand hygiene practices among indian medical undergraduates: a questionnaire-based survey. Cureus. 2017;9(7):1463.

14. Guidance for Industry. Fact sheet: Services USD of H and $\mathrm{H}$, administration $\mathrm{F}$ and $\mathrm{D},(\mathrm{CDER}) \mathrm{C}$ for $\mathrm{DE}$ and $\mathrm{R}$; temporary policy for preparation of certain alcoholbased hand sanitizer products during the public health emergency (COVID-19. Available at: https://www.regulations.gov. Accessed on 28 January 2021.

15. Ikhlaq A, Bint-E-riaz H, Bashir I, Ijaz F. Awareness and attitude of undergraduate medical students towards 2019-novel corona virus. Pak J Med Sci. 2020;36:32-6.

16. Meghna Pandey, Poonam Salwan, Juhi Singla SS and SR. Study and comparison of knowledge of medical students and interns about pharmacotherapeutics. Int J Adv Res. 2018;6(2):94-9.

17. Maheshwari S, Gupta P, Sinha R, Rawat P. Knowledge, attitude, and practice towards coronavirus disease 2019 (COVID-19) among medical students: a cross-sectional study. J Acute Dis. 2020;9(3):100.

18. Singh A, Dixit A, Kumar A, Pandey D, Singh C, Pathak A. A cross-sectional study to compare knowledge and perception of generic medicine among medical students at a tertiary care center. Natl $\mathbf{J}$ Physiol Pharm Pharmacol. 2020;10(0):1.

19. Desai M, Panchal J, Shah S, Iyer G. Evaluation of impact of teaching clinical pharmacology and rational therapeutics to medical undergraduates and interns. Int J Appl Basic Med Res. 2016;6(3):205.

Cite this article as: David R, George DM, Chandra S, Marwaha V, Palatty PL. Final year medical students versus interns: information seeking behaviour about COVID-19 therapy in India. Int $\mathbf{J}$ Basic Clin Pharmacol 2021;10:500-6. 